\title{
Natural variability in hard-bottom communities and possible drivers assessed by a time-series study in the SW Baltic Sea: know the noise to detect the change
}

\author{
M. Wahl, H.-H. Hinrichsen, A. Lehmann, and M. Lenz \\ GEOMAR Helmholtz Centre for Ocean Research, Duesternbrookerweg 20, 24105 Kiel, Germany \\ Correspondence to: M. Wahl (mwahl@geomar.de)
}

Received: 29 January 2013 - Published in Biogeosciences Discuss.: 18 February 2013

Revised: 12 June 2013 - Accepted: 25 June 2013 - Published: 30 July 2013

\begin{abstract}
In order to detect shifts in community structure and function associated with global change, the natural background fluctuation in these traits must be known. In a $6 \mathrm{yr}$ study we characterized the composition of young benthic communities at 7 sites along the $300 \mathrm{~km}$ coast of the Kiel and Lübeck bights in the German Baltic Sea and we quantified their interannual variability of taxonomic and functional composition.

Along the salinity gradient from NW to SE, the relative abundance of primary producers decreased while that of heterotrophs increased. Along the same gradient, annual productivity tended to increase. Taxonomic and functional richness were higher in Kiel Bight as compared to Lübeck Bight. With increasing species richness functional group richness showed saturation indicating an increasing functional redundancy in species rich communities.

While taxonomic fluctuations between years were substantial, functionality of the communities seem preserved in most cases. Environmental conditions potentially driving these fluctuations are winter temperatures and current regimes. We tentatively define a confidence range of natural variability in taxonomic and functional composition a departure from which might help identifying an ongoing regime shift driven by global change. In addition, we propose to use RELATE, a statistical procedure in the PRIMER (Plymouth Routines in Multivariate Ecological Research) package to distinguish directional shifts in time ("signal") from natural temporal fluctuations ("noise").
\end{abstract}

\section{Introduction}

The structure of communities, i.e. the number, identity and functional characteristics of the constituent species, determines both their ecosystem functions and their stability or resilience over time (e.g. Hillebrand, 2004; Solan et al., 2006; Harley et al., 2006; and Gamfeldt et al., 2008). Traits considered important characteristics of functional groups include body size, motility, growth form, reproductive mode, trophic type and modularity (e.g. Micheli and Halpern, 2005; Bremner et al., 2006a; and Wahl et al., 2011a). In many regions in all oceans, the structure of benthic communities is currently in a process of transformation (e.g. Cowie, 2007) as a consequence of global change (e.g. Harley et al., 2006). Main drivers of this re-structuring are warming, acidification, desalination, hypoxia, sea level rise and/or bioinvasions (e.g. Jackson and McIlvenny, 2011; Lockwood and Somero, 2011; Merzouk and Johnson, 2011; and Philippart et al., 2011). The proximate responses to these drivers are shifts in the behaviour and performance of organisms, changes in the relative abundance of species, local extinctions, range shifts and the ensuing re-structuring of communities (e.g. Gardner et al., 2011; O'Connor et al., 2007; and Harley et al., 2006). Species are the bearers of ecological traits which determine their performance, their persistence, their function and, ultimately, the services of a community (Millennium Ecosystem Assessment, 2005; Bremner et al., 2006a, b). The loss or gain of species may go along with the loss or gain of an ecological function if the species considered is its sole bearer in a given community. In contrast, functional shifts of a community may be dampened when more than one species bear a given functional trait 
(e.g. Wahl et al., 2011a) and when species' shifts are not too synchronous (Loreau and de Mazancourt, 2008). Since almost 5 decades, a link between species richness and community functioning and resilience has been repeatedly postulated ("insurance hypothesis", von Bertalanffy, 1960; McNaughton, 1977; Yachi and Loreau, 1999). If such a mechanistic connection exists, species-poor environments such as the Baltic Sea (Ojaveer et al., 2010) may be particularly sensitive to the stress imposed by Climate Change and local stressors (e.g. Frid, 2011; and Havenhand, 2012).

Climate variability is known to produce strong signals in benthic communities regarding structure, diversity and productivity (e.g. Pollack et al., 2011). Warming, in particular, affects physiological rates, phenology and body size of organisms (e.g. O'Connor et al., 2007; Somero, 2010; and Gardner et al., 2011) producing strong ecological (restructuring) and evolutionary responses in the benthos (e.g. Harley et al., 2006). Both climate variability and climate change rates may be particularly strong in the Baltic region (e.g. Lehmann et al., 2011; and BACC Author Group, 2008), making it a paramount requirement in this ecosystem to distinguish noise (fluctuations) from signal (climate change). As "noise" we consider a non-directional, interannual dissimilarity in community composition which is mainly driven by environmental fluctuations, "lottery effects" and stochasticity (see below). As "signal" we would consider a gradual and directed (i.e. associated with increasing dissimilarity with time) or an abrupt (regime shift) change in community composition presumably driven by long-term trends in environmental change (e.g. warming and eutrophication).

The drivers of structural change in communities may affect the species directly or via a shift in biological interactions (Kordas et al., 2011; Wahl et al., 2008) or specific phenologies (e.g. Sommer et al., 2012). Furthermore, hydrographical changes can lead to altered distribution patterns. While the potential risk of this large-scale, anthropogenic re-shuffling of benthic communities is widely recognized, detecting this change is difficult against the local background noise without adequate monitoring (Firth and Hawkins, 2011).

Natural fluctuations in the composition of benthic communities incorporate deterministic and stochastic elements (e.g. Frid et al., 1996; and Spencer et al., 2011). The former include yearly rhythms reflecting the seasonal alteration of ephemeral species or decadal rhythms such as North Atlantic Oscillation (NAO) cycles. The latter comprise the random occurrence of disturbances/recovery cycles and the lottery effect of founder species identity (e.g. Greene and Schoener, 1982; and Reise, 1991).

Assessing the background fluctuations is particularly important in species-poor, marginal systems like the Baltic Sea, because (i) abiotic variability is pronounced due to the small size of the water body, to the highly variable wind-driven current system (Lehmann et al., 2011) and to the reduced buffer capacity of brackish water (Thomsen et al., 2009, 2010; BACC author group, 2008), and because (ii) low species rich- ness - especially when combined to low functional richness makes communities more vulnerable to environmental shifts (e.g. Wahl et al., 2011a), since functional gaps caused by the decline of a species cannot always be filled by a functionally equivalent species (e.g. Frid, 2011).

The shallow water, hard-bottom communities in the western Baltic develop on granite boulders in an otherwise predominantly soft bottom habitat. Typically, in the course of a year some 60 sessile macroscopic species can be found on shallow (1-3 m depth) hard substrata (own unpublished data) of which roughly half are macroalgae, the other half suspension feeders. These species represent 15 functional groups according to a classification scheme recently proposed (Wahl et al., 2011), of which 11 are represented by 3 or less species and 5 just by one species (this study). According to the insurance hypothesis the functions combined in these 11 functional groups may be particularly threatened by species loss (Frid, 2011).

Monitoring of marine habitats has been regarded as oldfashioned for over 2 decades, but experiences a revival due to the recognition of its importance for global change evaluation (e.g. Anderson et al., 2012; and Carr et al., 2011). We can only decide whether a shift in species combination is attributable to global change, when we are able to distinguish it from the background noise, i.e. natural variability in species abundance. Temporally repeated sampling for monitoring purposes often includes an element of spatial or methodological heterogeneity when the collection is not carried out at the identical spot and/or by the same method. In these cases procedural, spatial and temporal variability in community composition may mask or amplify each other. Additional variance is added when 2 monitoring events sample two communities of different age, representing different stages in a successional process or are carried out in different seasons. In an effort to disentangle these different sources of variability and to minimize artefactual variability, we have established a series of fixed monitoring stations along the German Baltic coast, where replicate communities that develop for exactly $1 \mathrm{yr}$ on identical artificial substrata in the same water depth are collected every year in the same season. In this manner, we strive to analyse temporal fluctuation with a yearly resolution independently of other sources of variability.

Given this experimental set-up, the variability in community traits such as species composition or productivity is mainly driven by environmental factors such as temperature, salinity, nutrient concentrations, irradiation, intensity and quality of predation, and currents as vectors of resources and propagules. In this article we try to quantify (i) the spatial variability of species composition among the stations at the scale of $10 \mathrm{~s}$ of $\mathrm{km}$, (ii) the temporal year-to-year fluctuation of species composition within the stations and (iii) the associated shifts in community functioning, as well as (iv) the species and (v) the abiotic factors contributing most to these fluctuations. We tentatively define an interval of confidence within which compositional fluctuations of western Baltic 
Table 1. Coordinates and habitat characteristics of the stations sampled during this survey.

\begin{tabular}{|c|c|c|c|c|}
\hline Name & Code & $\mathrm{N}$ & E & Dominant habitats \\
\hline Falshöft & P1 & $54^{\circ} 46.935^{\prime}$ & $09^{\circ} 57.325^{\prime}$ & $\begin{array}{l}\text { sandy ground with dense stands of the sea } \\
\text { grass Zostera marina }\end{array}$ \\
\hline Boknis Eck & P2 & $54^{\circ} 32.942^{\prime}$ & $10^{\circ} 01.788^{\prime}$ & boulders covered with diverse macroalgae \\
\hline Schönberg & P3 & $54^{\circ} 24.272^{\prime}$ & $10^{\circ} 27.047^{\prime}$ & sandy with dense sea grass stands \\
\hline Fehmarn-NW & P4 & $54^{\circ} 32.147^{\prime}$ & $11^{\circ} 04.621^{\prime}$ & sandy without macrophytes \\
\hline Staberhuk & P5 & $54^{\circ} 24.096^{\prime}$ & $11^{\circ} 17.798^{\prime}$ & $\begin{array}{l}\text { mixed sand and boulder ground with sea- } \\
\text { grass on the former and blue mussels } \\
\text { Mytilus edulis on the latter }\end{array}$ \\
\hline Kellenhusen & P6 & $54^{\circ} 11.803^{\prime}$ & $11^{\circ} 05.901^{\prime}$ & with very large and dense mussel beds \\
\hline Salzhaff & P7 & $54^{\circ} 02.372^{\prime}$ & $11^{\circ} 31.559^{\prime}$ & $\begin{array}{l}\text { muddy ground with mixed macrophyte } \\
\text { communities dominated by the sea- } \\
\text { grass Zostera marina and the pondweed } \\
\text { Potamogeton sp. }\end{array}$ \\
\hline
\end{tabular}

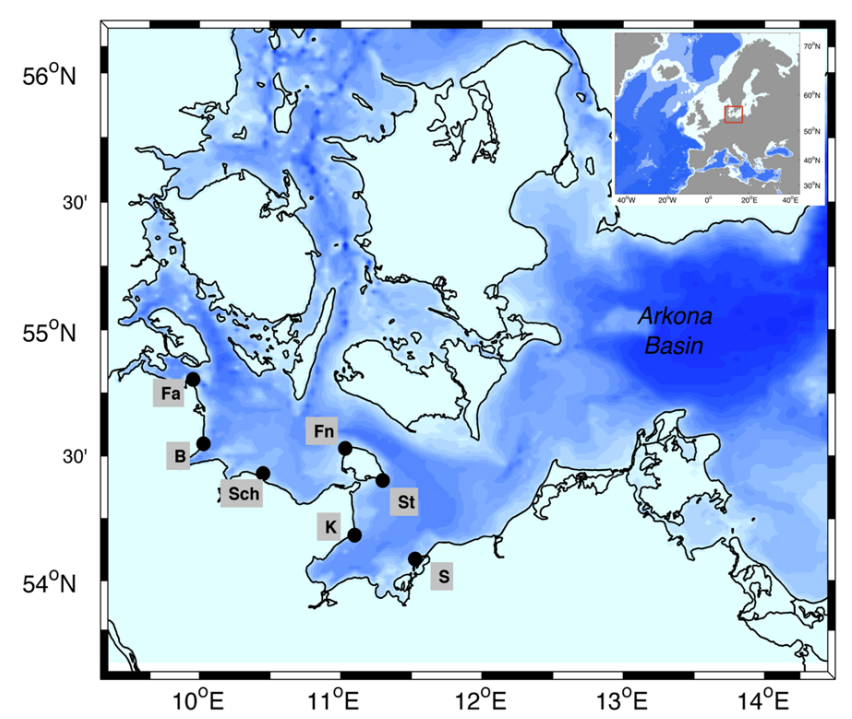

Fig. 1. Chart depicting the position of the seven stations in Kiel and Lübeck Bight sampled in this survey.

benthic communities are presumably "natural", whereas outside, which they could be, indicative of an anthropogenic signal (e.g. intensifying pollution) or climate change. This zone of confidence will become more reliable when the duration of the monitoring grows. Additionally, we recommend a known statistical procedure, RELATE, to distinguishing between directional shift in time and random temporal fluctuations in community composition. We will discuss the risk of overlooking change if the monitoring phase is started too late (or run for too long) and already includes first shifts in community structure driven by environmental change.

\section{Materials and methods}

\subsection{Assessment of community variability}

\subsubsection{Stations}

Along the German Baltic Sea coast, in the Kiel and Lübeck bights, seven permanent stations were selected for this longterm programme to assess hard-bottom community assemblage dynamics (Fig. 1). The stations from NW to SE were Falshöft, Boknis Eck, Schönberg, Fehmarn, Staberhuk, Kellenhusen and Salzhaff (see Table 1 for site characteristics). Among all stations, only Fehmarn is exposed to the regionally most frequent strong winds from the SW to the NW. The remaining sites are exposed to the rarer storms from northern or easterly directions. The dominant current directions and speeds are given in Fig. 2.

\subsubsection{Deployment of settlement panels}

At each station, eight $50 \mathrm{~cm} \times 50 \mathrm{~cm}$ concrete slabs were deployed at $3 \mathrm{~m}$ depth, with the distance between slabs being approximately $5 \mathrm{~m}$. Each slab was equipped with 2 vertical threaded stainless steel bars of $30 \mathrm{~cm}$ length. Each of these bars carried one horizontally orientated settlement panel $(12 \mathrm{~cm} \times 12 \mathrm{~cm})$ made of grey PVC roughened with sand paper (grade 60). The panels were fixed $30 \mathrm{~cm}$ above ground with stainless steel nuts on either side. Panels were exchanged for new ones every September for the assessment of interannual variability in recruitment and community assemblage over the previous 12 months. The panels were harvested by unscrewing the upper nut of the support bar and transferring each panel individually into one ziplock bag already under water (SCUBA). Onboard a research vessel, the communities were fixed by adding buffered formalin (final concentration $4 \%$ ) to each ziplock bag. 
$\mathrm{Fa}$

B Sch
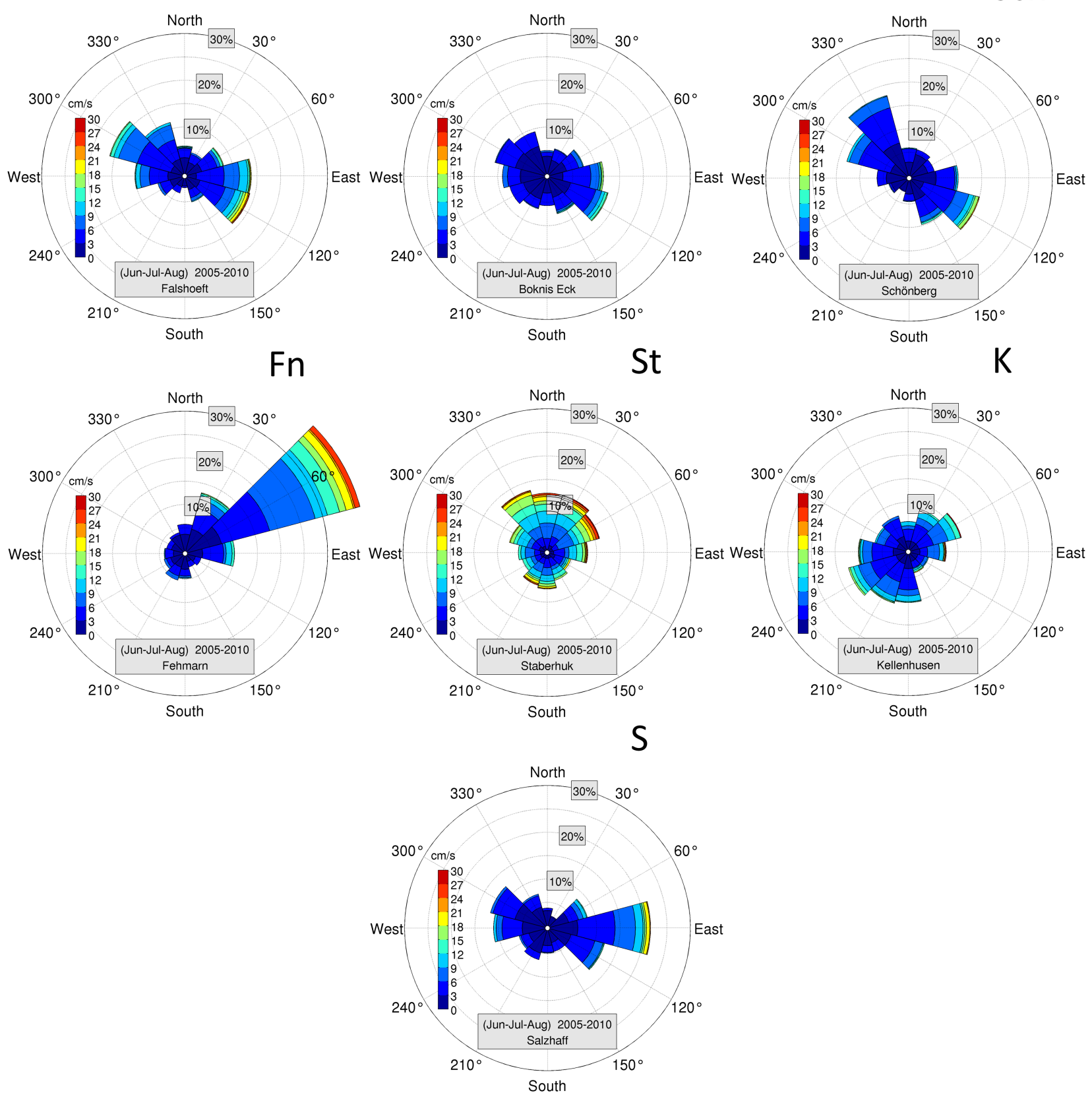

Fig. 2. Distribution of current regimes at the seven stations, averaged over all six years of the survey. Degrees indicate the current directions (by segments of $30^{\circ}$ ), percentages give the commonness of a given current direction, the colour coding indicates the average speed of currents in a given directional segment. (A) through $(\mathbf{G})$ are the summer current regimes at the different stations Fa through $\mathrm{S}$.

\subsubsection{Characterization of communities}

In the lab, the various components of the communities were identified to the lowest possible taxonomic level (species or genus), and their relative cover on the panel was estimated to the nearest $5 \%$. Additionally, each genus or species was attributed to a functional group defined by the adult properties with regard to size, growth form, trophic type and modularity.
Each species is attributed a 4-letter code that identifies which of the 16 traits in the 4 metrics apply (Table 2). This code is believed to define an ecological role of a species well (e.g. Wahl et al., 2011a). Thus, a barnacle (MMSS) ecologically resembles more closely a mussel (MMSS) than a hydrozoan (LBSC) or an encrusting red alga (MEAS). The functional characterization allowed assessing whether structural change in communities was accompanied by functional change. It 
Table 2. Functional traits used for grouping. The metrics used are considered ecologically relevant and they can be surrogates for other traits. Thus, body size correlates closely with longevity or metabolic rate, growth form determines the species' strategy for exploiting resources such as substratum or light, etc. Ecosystem services associated with these and similar traits are discussed in Bremner et al. (2006) and Wahl (2009).

\begin{tabular}{llll}
\hline Adult body size & Growth form & Trophic type & Modularity \\
\hline S $<1 \mathrm{~mm}$ & E encrusting & A autotroph & S solitary \\
M $1-10 \mathrm{~mm}$ & M massive & P predator & C colonial \\
L $10-100 \mathrm{~mm}$ & B bushy & S suspension feeder & \\
XL $100-1000 \mathrm{~mm}$ & F filamentous & D deposit feeder & \\
XXL $>1000 \mathrm{~mm}$ & & G grazer & \\
\hline
\end{tabular}

should be noted that this functional characterization applies to the adult stage only.

Subsequently all organisms were scraped off the panel and dried to constant weight at $60^{\circ} \mathrm{C}$ to obtain the community dry weight, then burned at $500^{\circ} \mathrm{C}$ for $24 \mathrm{~h}$ to obtain the community ash weight.

Species or genus identity as well as relative abundance were used to describe and analyse the compositional properties of a panel community. Dry weight (DW) and ash free dry weight (AFDW) were obtained to describe the panel communities' productivity as biomass accumulation over 12 months. While community composition was assessed only on the upward facing side of the panel to also include all primary producers, biomass was recorded from both panel sides and was standardized per $100 \mathrm{~cm}^{2}$.

\subsubsection{Statistics for community analysis}

Data processing: the majority of analyses presented in this study, except the assessment of community biomass, were exclusively performed on the biota that established on the upward facing surface of the settlement panels. Furthermore, we only considered sessile and hemi-sessile (such as tubedwelling gammarids of the genus Corophium) species and ignored all components of the associated motile fauna, which were not part of a fouling ("attached") community sensu stricto and that are not being caught quantitatively in the ziplock bags used for sampling. Since not all of the organisms encountered on the panels could be identified on the species level, the genus was used as the level of taxonomic resolution for all analyses.

Data analyses: correlations between single variables were either Pearson's (if data were normal) or Spearman's rank (if data were not normal) correlations. All multivariate analyses were done with the PRIMER (Plymouth Routines in Multivariate Ecological Research) 6.0 software package (Clarke and Warwick, 2007; Clarke and Gorley, 2006). We used principal component analysis (PCA) to identify the most relevant species, i.e. those that were responsible for the bigger part of the temporal and spatial variation in community structure during the observation period. For this, only genera which showed $\geq 10 \%$ cover on at least one of the sampled settlement panels were selected. PCA was performed on the untransformed data with a maximum of 5 principal components. We tested for linear relationships between variables using pair plots prior to this analysis and found no tight, nonlinear correlation between any of the species.

To assess the temporal (year to year) dynamics in community composition at the seven study sites, we calculated either the similarity (for the MDS plots) or the dissimilarity (for the variability graphs and the SIMPER (similarity percentage) analysis) between assemblages that established at the same location in different years. For this, species abundance data were averaged across all 8 replicates that were sampled at each site in $1 \mathrm{yr}$, and then the Bray-Curtis (dis) similarity for all of the 35 possible pairings ( 7 sites, $6 \mathrm{yr}$ ) was calculated on the basis of these data. In order to describe a confidence interval of "natural" interannual dissimilarity in community structure at a given site, we calculated the mean dissimilarity between communities of year $x$ and of year $y$ at a given site. Then we calculated the average and $95 \%$ confidence interval of dissimilarity between communities of 2 different years at this site over the entire monitoring period. This provided us with the information about the mean and spread (CI) of interannual fluctuations in community composition for all the monitoring sites over all the monitoring period. We proceeded in an analogous manner for the average and CI in the functional composition of communities. We also tested for the presence of a serial pattern in community change over time (directional shift) using the RELATE procedure in PRIMER (Clarke and Gorley, 2006; Clarke and Warwick, 2007). For these analyses, data were not transformed nor standardized. SIMPER analyses quantified the contributions of single species to the dissimilarity between a given pair of communities.

We ran simple linear regressions (if residuals were normal) or Spearman's rank correlations (if residuals were not normal) for each month of the year to elucidate the relationship between the abundances of the species that were identified by PCA as the most relevant ones in our study system, i.e. Mytilus edulis, Amphibalanus improvisus and Polysiphonia sp. (sampled in September of each year), and the average monthly temperatures (derived from the hydrodynamic model described earlier) of the preceding 12 months (from September of the previous year to August of the same year) in order to identify potential drivers of recruitment patterns. For the analysis, we pooled all data without stratifying them by station or year. Abundance and biomass data were averaged across all replicates that we sampled at a given station in a given year. We tested for normality of errors as well as homogeneity of variances using the graphical diagnostics provided by the free statistical computing software R (R Development Core Team, 2010). 
Table 3. Taxonomic and functional richness at the 7 stations: alpha richness is the mean number of taxa or functional groups per panel, gamma richness is the total number of taxa or functional groups found on all panels of a given station (averaged over the years). SD: standard deviation.

\begin{tabular}{lrrrrrrrr}
\hline & \multicolumn{3}{c}{ Species Richness } & \multicolumn{3}{c}{ Functional Group Richness } \\
& \multicolumn{2}{c}{ Alpha } & \multicolumn{2}{c}{ Gamma } & \multicolumn{2}{c}{ Alpha } & \multicolumn{2}{c}{ Gamma } \\
\cline { 2 - 10 } & Mean & SD & Mean & SD & Mean & SD & Mean & SD \\
\hline Falshöft (P1) & 6.0 & 2.7 & 11.0 & 3.7 & 3.7 & 1.1 & 6.0 & 0.6 \\
Boknis Eck (P2) & 6.0 & 2.6 & 10.5 & 2.5 & 4.0 & 1.6 & 5.3 & 1.6 \\
Schönberg (P3) & 5.5 & 2.4 & 11.0 & 2.1 & 3.8 & 1.3 & 6.2 & 1.5 \\
Fehmarn-NW (P4) & 5.8 & 2.2 & 12.5 & 3.6 & 4.2 & 1.4 & 7.3 & 1.9 \\
Staberhuk (P5) & 5.0 & 2.0 & 10.2 & 3.0 & 3.5 & 1.2 & 6.0 & 1.5 \\
Kellenhusen (P6) & 3.6 & 1.8 & 7.0 & 2.3 & 2.5 & 1.2 & 4.5 & 1.4 \\
Salzhaff (P7) & 4.6 & 2.0 & 9.2 & 2.7 & 3.5 & 1.3 & 5.7 & 1.8 \\
\hline
\end{tabular}

\subsection{Environmental variability}

\subsubsection{Hydrodynamic model}

To provide habitat information in terms of temperature, salinity and ocean currents, a comprehensive database was created containing the temporal development of the hydrographic conditions at each of the individual stations (Fig. 1). For this purpose, a three-dimensional hydrodynamic model was used to simulate the hydrographic conditions and their variations in space and time (Kiel Baltic Sea Ice-Ocean ModelBSIOM; Lehmann and Hinrichsen, 2000; Lehmann et al., 2002). The model domain comprises the entire Baltic Sea including the Gulf of Bothnia, Gulf of Finland, Gulf of Riga as well as the Belt Sea, Kattegat and Skagerrak. The horizontal resolution of the coupled sea ice-ocean model is at present $2.5 \mathrm{~km}$, with 60 vertical levels specified. The coupled sea ice-ocean model is forced by realistic atmospheric conditions taken from the Swedish Meteorological and Hydrological Institute (SMHI Norrköping, Sweden) meteorological database (L. Meuller, personal communication, 2003) which covers the whole Baltic drainage basin on a regular grid of $1^{\circ} \times 1^{\circ}$ with a temporal increment of $3 \mathrm{~h}$. The database consists of synoptic measurements interpolated on the regular grid by using a 2-D uni-variate optimum interpolation scheme. This database, which for modelling purposes is further interpolated onto the model grid, includes surface pressure, precipitation, cloudiness, air temperature and water vapour mixing ratio at $2 \mathrm{~m}$ height and geostrophic wind. Wind speed and wind direction at $10 \mathrm{~m}$ height are calculated from geostrophic winds in consideration of different roughness on the open sea and near the coastal area (Bumke et al., 1998). Forcing functions of BSIOM such as wind stress, radiation and heat fluxes were calculated according to Rudolph and Lehmann (2006). Physical properties simulated by the hydrodynamic model agree well with known circulation features and observed physical conditions in the Baltic Sea (e.g. Hinrichsen et al., 1997; and Lehmann et al., 2012).

\subsubsection{Drift model}

Simulated three-dimensional velocity fields were extracted from daily mean property fields provided by the hydrodynamic model in order to develop a database for particle tracking. This data set offers the possibility to derive Lagrangian drift routes by calculating the advection of "marked" water particles through space and time. The three-dimensional trajectories of the simulated drifters were computed using a 4th order Runge-Kutta scheme (Hinrichsen et al., 1997). In order to consider the particle drift in relation to its spatial and temporal variability, particles were seeded for the time period 2005-2009 at the panel locations every 5 days from 1 July to 30 August, resulting in 13 different release dates. The latter is based on the duration of the maximum dispersal and recruitment of many locally abundant species (Thomsen et al., 2010).

The experimental design required the determination of the origins of individual particles which settled during the prime settlement period (late May to August, Thomsen et al., 2010) at the panel positions (Fig. 1). Thus, particles were inserted into the simulated flow fields at the sea surface at the different panel positions and their drift was back calculated for a 40 days drift period. For each of the different particle release dates, 175 drifters were seeded on a regular spaced grid around the panel positions. The employed particle tracking technique allowed backward calculation of drift trajectories by simply reversing the temporal sequence of the three-dimensional flow fields and by inverting the sign of the horizontal components of the velocity vector (Hinrichsen et al., 1997).

\subsubsection{Statistical parameters of original particle distributions}

The model runs described in the previous section proved to be sufficient to determine the potential starting positions of particles which were calculated backward in time for a 40 days drift period from the panel positions. The 
characterisation of the back-calculated starting positions of the particles was performed by the calculation of statistical parameters including PCA. In our case, PCA has been used in a simple direction to determine the first two principle components from a bivariate data set with latitudes and longitudes of the starting positions of the particles. The spatial extension and density of the starting positions of the particles is defined as the dispersal kernel (Edwards et al., 2007). By using this approach, we have quantitatively estimated the extension of the particle release area by calculating their dispersal kernels (Edwards et al., 2007) in terms of variance ellipses. The calculation is based on the variance of the spatial components (longitudes and latitudes of the starting positions of the particles) as well as on their covariance. The latter enables us to find a principle angle describing the variations of particles around a mean position along a set of directions other than those of the longitudinal and latitudinal axis of the particle distributions (Preisendorfer, 1988). The dispersal kernels were found to be Gaussian in form, and similar to Edwards et al., (2007) we calculated four parameters: (1) the mean of the starting positions of particles, (2) the major and (3) minor axis of variability of the spatial starting positions of particles and (4) the principal angle of the orientation of the major axis. The identification of the dispersal scale (extension of the particle distribution) is given by the size of the variance ellipse as calculated from the major and minor axes sizes.

Variability of the sea surface environmental variables such as current direction, current velocity, salinity and temperature were quantified as the mean Bray-Curtis dissimilarities between consecutive years.

\subsubsection{Relation between hydrographical and compositional variability}

For the assessment of the influence of the interannual variability in hydrographical conditions (see below) during the period of maximum recruitment (June to August) on the interannual variability in community composition the oceanographic variables used were the June-July-August-averages in sea surface salinity, temperature, current velocity and current direction at each station. Main current velocities and directions were site dependent, thus the recruitment depends not only on the interannual variability of the atmospheric conditions but also on the site characteristics (Fig. 1). Histograms of daily surface velocities distributed over $30^{\circ}$-segment velocity directions were extracted from BSIOM for the period 2005-2010 (Fig. 2). Those sites where the flow is of retentive character show small current velocities and nearly equal distribution of current directions (e.g. B and K); those which are of dispersive character show stronger current velocities and specific current directions (e.g. Sch and Fn). It should be noted that for Salzhaff, currents would most probably be overestimated because of the limited model resolution. Biological, i.e. differences in species abundances, as well as oceanographic dissimilari-

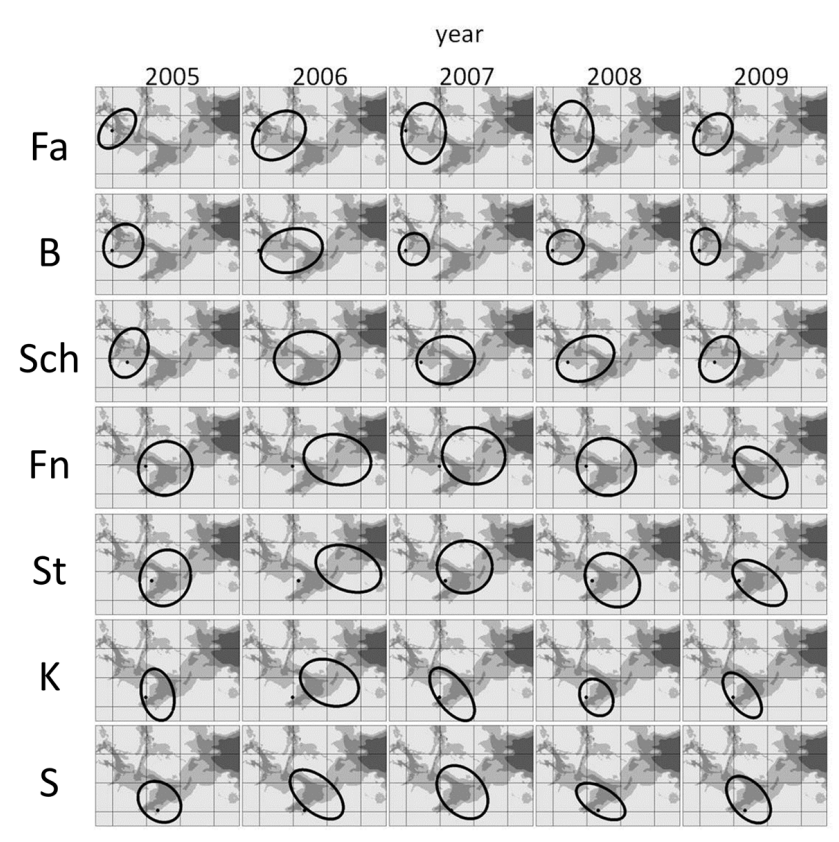

Fig. 3. Dispersal kernels describing the back-calculated origins of drifting particles released at the different platform locations (black dots), 1st to 7th Rows represent the locations FA = Falshöft, $\mathrm{B}=$ Boknis Eck, Sch = Schönberg, Fn = Fehmarn, St = Staberhuk, K = Kellenhusen, and $\mathrm{S}=$ Salzhaff.

ties, i.e. differences in salinity, temperature, current velocity and direction, between consecutive years were calculated for each site using PRIMER. Moreover, to come to one value for the oceanographic variables, the observed dissimilarities (all in \%) were averaged across all four of them. Biological and the averaged oceanographic dissimilarities between two consecutive years then formed the statistical pairs that were analysed graphically and by simple linear regression.

\section{Results}

\subsection{Site characteristics}

The stations differed with regard to their habitat characteristics (Table 1), their salinity, their current regime (Fig. 2) and the variability in hydrographic conditions (Figs. 3, 11). Salinity tended to decrease from NW to SE and current variability peaked in the central region around the island of Fehmarn. Generally, for the backward-calculated particles initially released at the different stations in the western Baltic (Fig. 1), stations differ substantially (Fig. 2a-g). The 3 western-most stations, Falshöft, Boknis and Schönberg feature mainly north-westerly and south-easterly currents of moderate speed. Fehmarn is characterized by a dominance of strong north-easterly currents, whereas the most eastern stations Staberhuk, Kellenhusen and Salzhaff experience weak to moderate currents in various directions. Sizes 


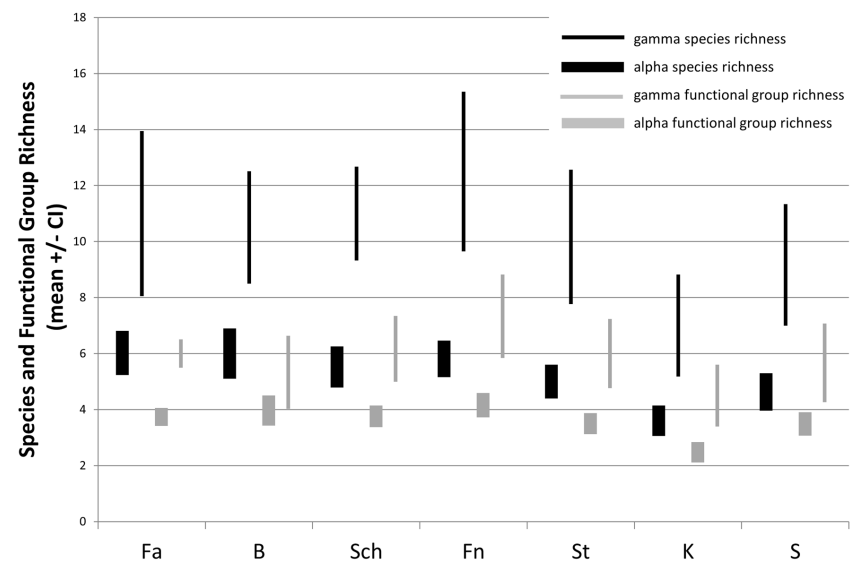

Fig. 4. Confidence intervals of richness in species and in functional groups at the panel (alpha diversity) and station level (gamma diversity). Here and elsewhere: B, Boknis Eck; Fa, Falshöft; Fn, Fehmarn; K, Kellenhusen; S, Salzhaff; Sch, Schönberg; St, Staberhuk.

and orientations of the variance ellipses indicate interannual variability of the potential backward-calculated origins of drifters as well variations among stations (Fig. 3). The largest horizontal extensions (ellipse area sizes) for most of the drifter origins occurred for the years 2006 and 2007, while the extensions in other years (2005, 2008 and 2009) are relatively small. Marked differences in drift patterns can be recognized when comparisons are made between simulations for the different stations. For the panel locations Falshöft, Boknis, Kellenhusen and Salzhaff, all drifters tended to originate from areas relatively small in size, indicating a general dominance of retention, i.e. the origin of particles were located relatively close to their final panel positions. The variance ellipses of the most north-western station Falshoeft yielded the highest variation in north-south direction, while for the stations Boknis to Staberhuk the orientation of the ellipses indicates extension of backward-calculated origin of drifters mainly varying in east-west direction. Different patterns emerged for the location Schönberg to Staberhuk, which could be characterized by dispersal. These locations are probably influenced to a higher degree by particles originating in the Lübeck and Mecklenburg Bight as well as in the Arkona Basin, likely as a consequence of currents induced by prevailing weak to moderate winds of eastern direction. Obviously, largest drift distances were obtained for those time periods and locations, when the stations were located outside the variance ellipses. The latter was mainly observed in 2006 at stations located east of $10^{\circ} \mathrm{E}$.

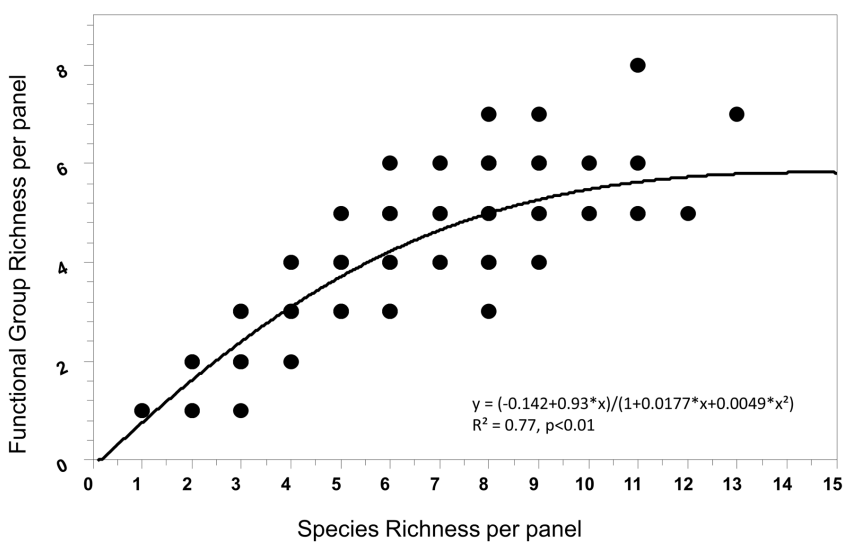

Fig. 5. Relation between species richness and functional group richness at the level of panels. Functional group richness shows saturation at elevated taxonomic richness indicating functional redundancy in diverse communities. Note that single dots may represent several replicates and $n=277$ panels.

\subsection{Community traits}

\subsubsection{Taxonomic and functional composition}

A total of 60 sessile species/genera were found in all stations and years. Communities in Kiel Bight (Falshöft through Fehmarn) tended to be more taxa-rich at the panel (mean alpha diversity around 6) as well as at the station level (mean gamma diversity around 11-12) as compared to Lübeck Bight (around 4 and around 8, respectively) (Fig. 4, Table 3). Similarly, the richness in functional groups tended to be higher in Kiel Bight (alpha diversity around 4, gamma diversity around 6) than in Lübeck Bight (alpha diversity around 3, gamma diversity around 5-6) (Fig. 4). Generally, the functional group diversity of panel communities increased with taxa diversity but approached saturation beyond a taxa richness of 9 (Fig. 5). This indicates that in taxa rich communities redundancy within functional groups was higher. Of the 60 taxa found, 11 were considered common, i.e. on average over all stations and years they contributed at least $1 \%$ to the panel coverage. These were the macroalgae Cladophora sp., Callithamnion sp., Dasya baillouviana, Hildenbrandia rubra, Ceramium spp. and Polysiphonia spp. and the sessile animals Electra pilosa (bryozoan), Corophium volutator (mud tube building amphipod), Polydora ciliata (mud tube building polychaete), Amphibalanus improvisus (barnacle) and Mytilus edulis (mussel) (Fig. 6). All taxa (hereafter called by their genus names) could, at least occasionally, be found at all stations. Some conspicuous trends, however, were apparent: Dasya, Cladophora and Amphibalanus tended to decrease from NW to SE, while Mytilus and Electra showed the opposite trend. Polysiphonia and Corophium were most abundant in the centre of the salinity gradient that the monitoring programme covers (Fig. 6). PCA identified the blue mussel Mytilus, the barnacle Amphibalanus and red algae of 


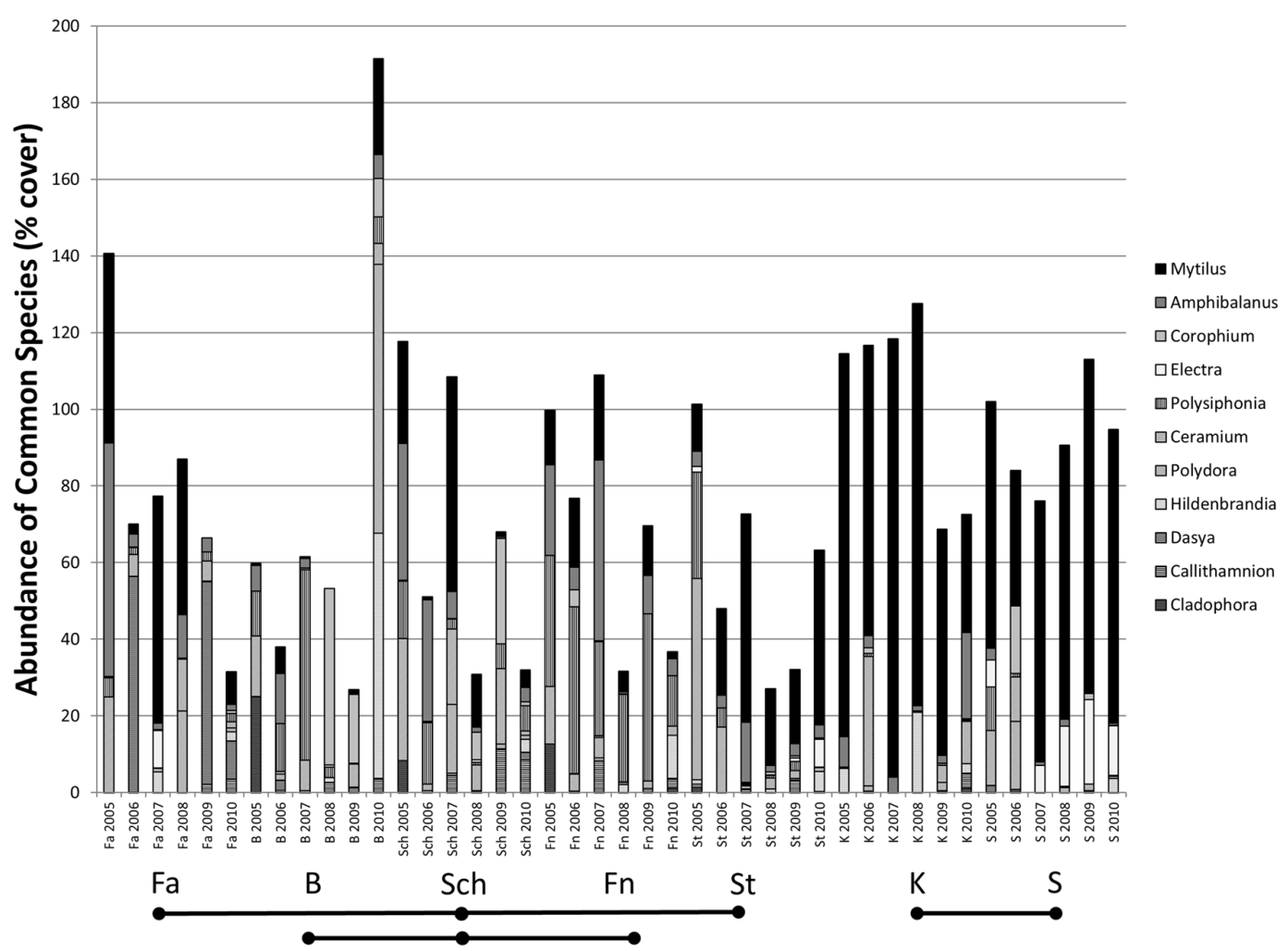

Fig. 6. Mean cover $(\%)$ of the more common species at the 7 stations and in the $6 \mathrm{yr}$ of surveillance. Station dots connected by lines at the base of the graph do not differ significantly in community composition.

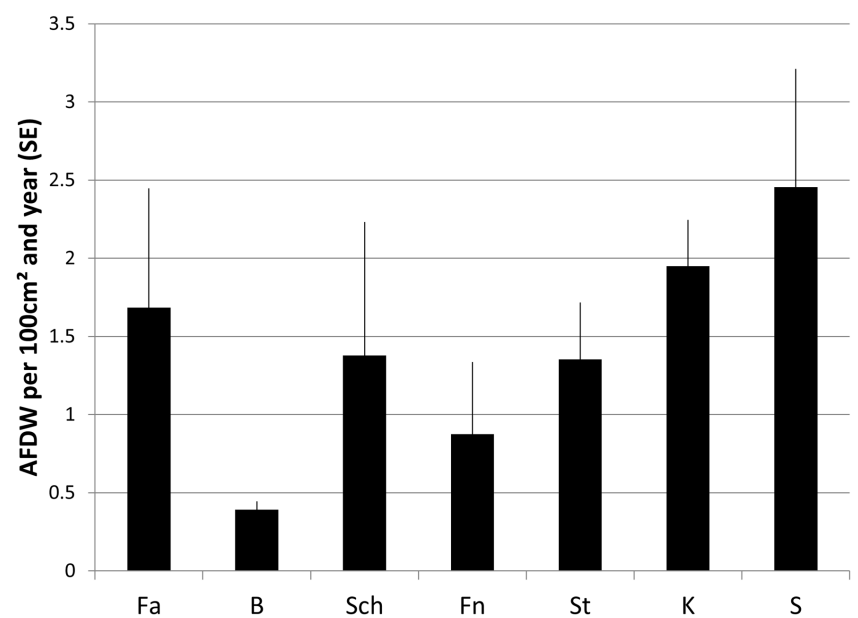

Fig. 7. Mean yearly productivity at the various stations expressed as ash free dry weight (AFDW) accrual per $100 \mathrm{~cm}^{2}$ and year.

the genus Polysiphonia as the ones that contributed most to temporal and spatial variability in community structure.

The 60 observed taxa belonged to 15 functional groups (as used in this study). Overall, 16 species were large, 28 medium and 16 small. A filamentous growth form was realized by 29 species, a massive growth by 25 and an encrusting growth by 6 species. 24 algal species and 26 animal species were assessed, of which 50 were solitary and 10 colonial. All of the animal species were suspension feeders since only sessile species were taken into account.

Within $1 \mathrm{yr}$ of recruitment, the upper sides of the panels were, on average, covered to between $57 \%( \pm 11.3 \%$ $\mathrm{SE})$ in Staberhuk and $103 \%( \pm 10 \% \mathrm{SE})$ in Kellenhusen. The two sites east of the isle of Fehmarn (Kellenhusen, Salzhaff) showed generally more cover than the sites on Fehmarn (Fehmarn, Staberhuk) and west of it (Falshöft, Boknis Eck, Schönberg, data not shown). Productivity expressed as accrual of ash free dry weight (AFDW) over 12 months tended to increase from NW to SE with the exception of the western-most site, Falshöft, were it was intermediate (Fig. 7). Lowest productivity was found in Boknis Eck and highest in Salzhaff with $0.39( \pm 0.05 \mathrm{SE})$ and $2.46( \pm 0.76 \mathrm{SE}) \mathrm{g}$ AFDW $100 \mathrm{~cm}^{-2} \mathrm{yr}^{-1}$, respectively. At the 3 western most stations Falshöft, Boknis Eck and Schönberg community productivity was realized to almost equal parts by heteroand autotrophs, but south-eastward from Fehmarn the contribution of heterotrophs continuously increased to reach over $90 \%$ in Salzhaff (Fig. 8). The production of AFDW correlated positively with the relative abundance of heterotrophs $(r=0.816, p<0.05)$. 


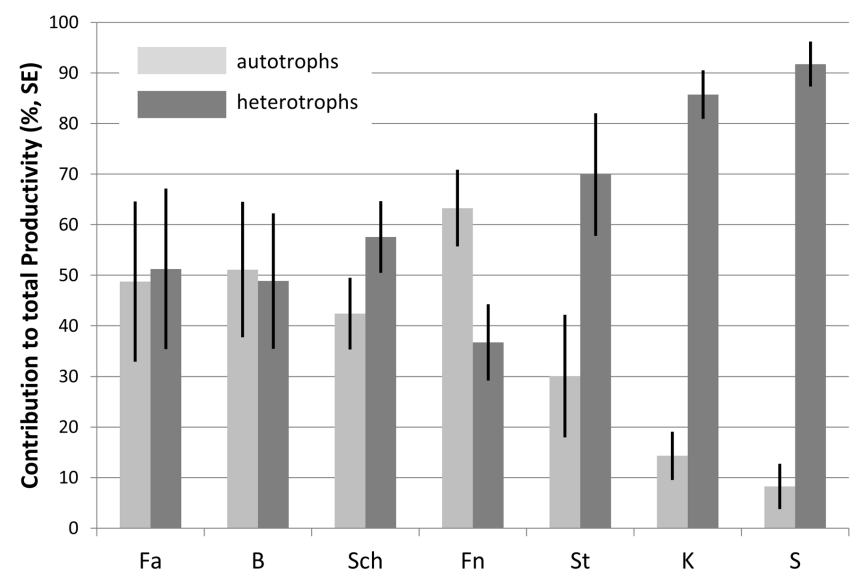

Fig. 8. Mean relative contribution of macroalgae (autotrophs) and sessile animals (heterotrophs) to the total annual productivity (\% cover per panel) of a panel community (averaged over all panels and years for each station).

\subsubsection{Interannual variability in community structure}

The communities that assembled on the panels of a given station over 12 months differed between consecutive years by 30 to $75 \%$ (Bray-Curtis dissimilarity based on genera richness). At most stations the interannual shifts in community structure (or function) do not seem to follow a directional trajectory, i.e. their dissimilarity to the initial structure in 2005 did not increase over time (Fig. 9a, b). In Kellenhusen the community composition was most stable over time. In Falshöft, Schönberg, Fehmarn and Salzhaff community composition seems to move randomly around some virtual centre. In contrast to this, a tendency towards a directional shift might be detected in Boknis Eck and Staberhuk (Fig. 9a). However, according to the RELATE procedure we employed to detect directional change in community structure over time, none of the trajectories shown in Fig. 9a, including Boknis Eck and Staberhuk, showed a significant temporal seriation. Falshöft seems to oscillate between a Dasya-dominated and a Mytilus-dominated status (Figs. 6, 9a). Boknis Eck shifted from a Cladophora/Ceramium community via a Corophium community towards a Hildenbrandia/Polydora community. Schönberg moved from a $\mathrm{Ce}$ ramium/Amphibalanus community via a Ceramium/Mytilus status towards a Corophium/Ceramium community. Fehmarn was in almost all years dominated by Polysiphonia and Amphibalanus, while communities in Staberhuk and Kellenhusen were always shaped by Mytilus. Only in Staberhuk we observed a shift in sub-dominant species from $\mathrm{Ce}$ ramium over Amphibalanus to Electra. Salzhaff started as a Mytilus/Amphibalanus community, showed an intermediate status with much Ceramium, Polydora and Corophium besides the dominant Mytilus and featured an even more marked Mytilus dominance with associated Electra in the last years (Figs. 6, 9a). Repeating the analysis with Bray-Curtis

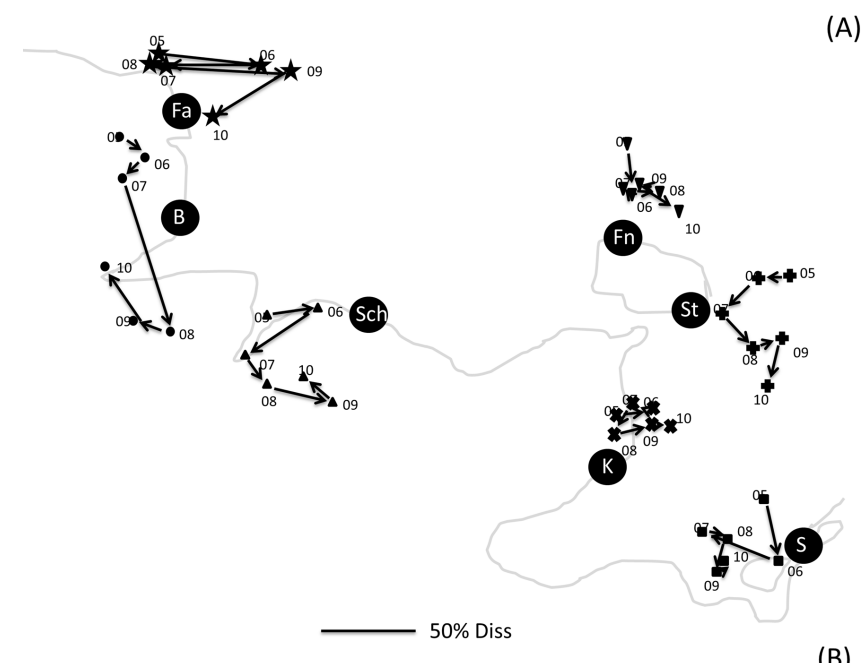

(B)

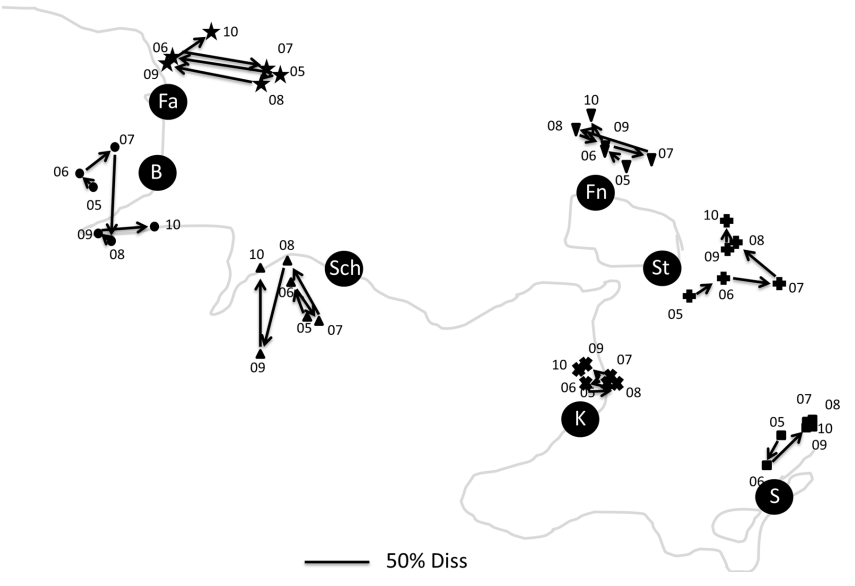

Fig. 9. MDS trajectories between consecutive years for taxonomic (A) and functional (B) composition of the panel communities. The station codes $(\mathrm{Fa}-\mathrm{S})$ are placed on the approximate position of the site relative to the coast line in light grey. Within, but not between, compositional categories (species (A), functional groups (B)) are all plots drawn to scale, i.e. same lengths and directions of trajectories correspond to an equivalent amount of dissimilarity between consecutive years. A scale bar indicating the $50 \%$ dissimilarity distance is given in each plot.

dissimilarity based on functional richness reveals similar patterns for the different stations (Fig. 9b) and again no indication for a significant directional change over time at any of the stations was detectable by RELATE. The shift patterns in taxonomic and functional composition of the communities behave quite similarly since the respective rho values of the RELATE analyses correlate positively $\left(R^{2}=0.77\right.$, $p<0.01)$.

Interestingly, the interannual variability in taxonomic composition decreased from NW to SE almost steadily from over $80 \%$ mean dissimilarity between subsequent years in Falshöft and Boknis Eck to below $60 \%$ in Kellenhusen and Salzhaff (Fig. 10). Only a handful of taxa were responsible for most $(71 \%)$ of all compositional variance (SIMPER 


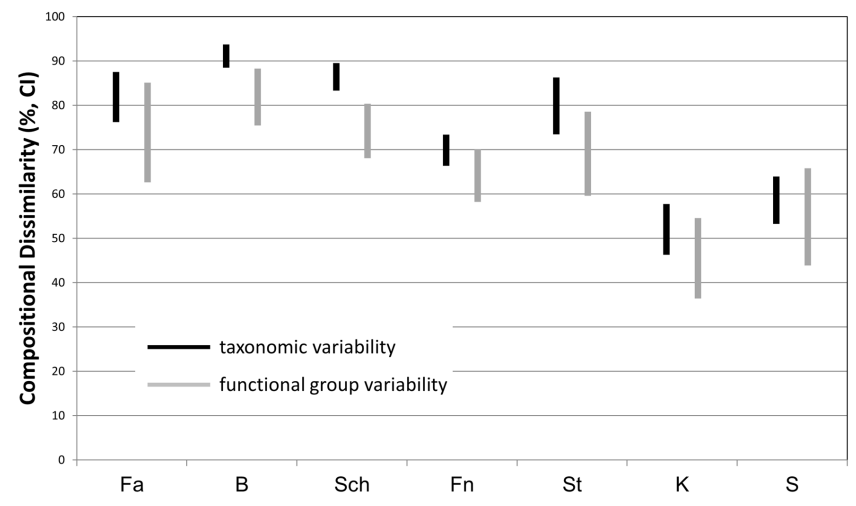

Fig. 10. $95 \%$ confidence intervals of interannual variability in taxonomic (dark grey bars) and functional composition (light grey bars). The confidence interval defines the zone of interannual variability considered the natural background "noise" (based on the $6 \mathrm{yr}$ surveillance).

analysis): Mytilus edulis (14\%), Amphibalanus improvisus (11\%), Ceramium spp. (11\%), Polysiphonia spp. (8\%), Polydora ciliata (7\%), Callithamnion sp. (6\%), Corophium volutator (6\%), Hildenbrandia rubra (5\%), and Dasya baillouviana (4\%). The compositional variability pattern was closely matched by a similar pattern in the variability of functional groups (Fig. 10). However, the most important functional traits (medium to large body size for biomass accrual and longevity; autotrophy for primary production; suspension feeding for pelago-benthic coupling, see Frid et al., 2008) were almost always represented redundantly in a panel community. Medium to large forms are represented by at least one species in all 277 communities, and redundantly so (by 2 or more species) in $98 \%$ of these communities. Suspension feeding is represented by at least one species in $97 \%$ of the communities and redundantly so in over $80 \%$ of these. Autotrophy is represented by at least one species in $90 \%$ of the communities and redundantly so in $72 \%$ of these.

\subsection{Potential drivers of compositional variability}

Total abiotic variability (i.e. Bray-Curtis dissimilarities of environmental data) between consecutive years tended to be higher in the western as compared to the eastern part of the range assessed (Fig. 11a) This was mainly due to differences in the variability of the current regime since salinity and temperature were quite similar in time (less than 7 and $5 \%$ dissimilarity between consecutive years). The modelled area of provenance of the propagules (Fig. 11b), i.e. the variance ellipse area sizes (see Fig. 3) representing the variability of current directions weighted by their velocity vector sizes, was most variable in the centre of the geographical range, i.e. around the island of Fehmarn (sites Fn and St) (Figs. 3, 11b). The mean abiotic dissimilarity between the summer months (June-August) of consecutive years (mean of the Bray-Curtis dissimilarities in current direction and veloc-

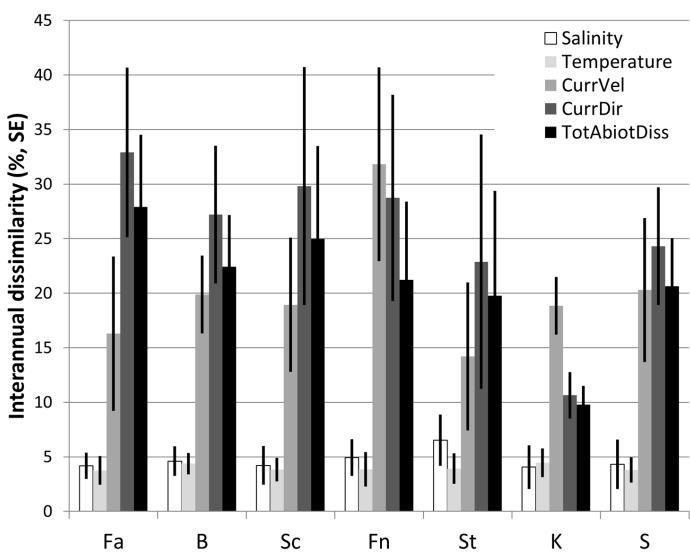

(A)

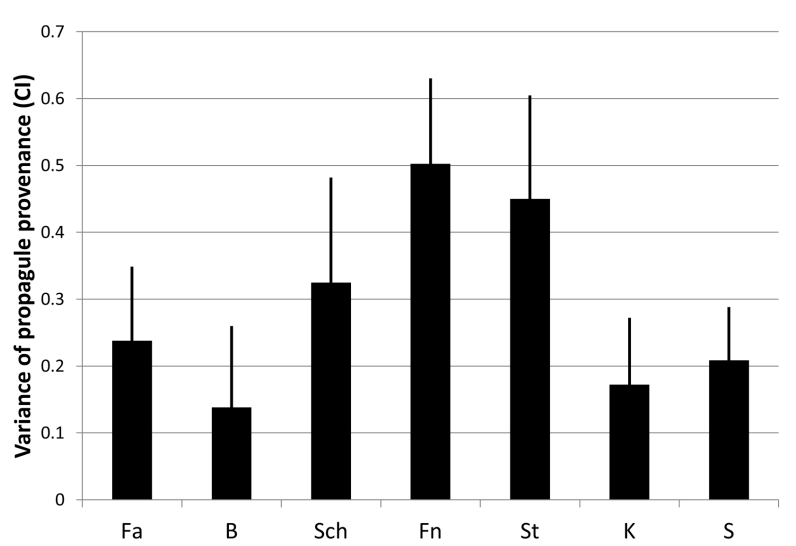

(B)

Fig. 11. (A) Mean dissimilarity between consecutive years in salinity, temperature, current velocity (CurrVel), current direction (CurrDir) and of all these four variables together (TotAbiotDiss) averaged over the months of June, July, August and over the $6 \mathrm{yr}$ of surveillance. (B) Variance of the propagule origin (area of the dispersal kernels of Fig.3) averaged over the $6 \mathrm{yr}$ of surveillance (with CI).

ity, salinity and temperature) correlated positively with the biotic dissimilarity between consecutive years (Bray-Curtis dissimilarity of species composition) (Fig. 12). However, due to the large variance, especially of the abiotic variables, this relationship was only marginally significant $(p=0.057)$, but abiotic variance explained about $55 \%$ of biotic variance.

The winter temperatures seemed to have some effect on the composition of the communities. The monthly mean temperatures of the preceding 12 months affected the 3 target species to variable degrees. Warm winters (November through February, with minimum water temperatures in February between 3 and $5^{\circ} \mathrm{C}$ ) favoured the barnacles weakly, but the mussels quite strongly, while both, warm winters and warm summers (with minimum water temperatures in July and August $\geq 20^{\circ} \mathrm{C}$ ), disfavoured the filamentous red algae (Fig. 13a-c). 


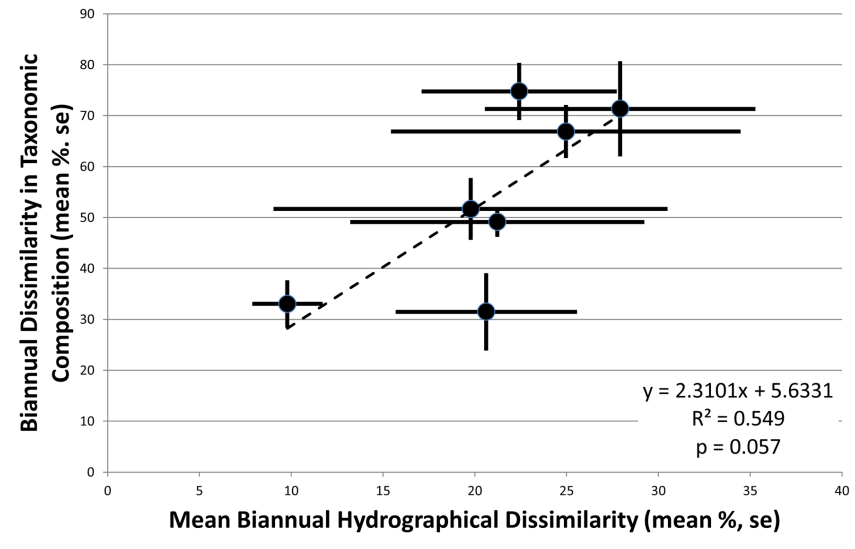

Fig. 12. Relationships (marginally significant) between taxonomical dissimilarity of communities between consecutive years and the dissimilarity of hydrographical conditions (direction and velocity of currents, salinity, temperature) among the same pairs of years.

Monthly mean salinity did not substantially affect the 3 target species, which drove the bulk of the biotic dissimilarity among consecutive years.

\section{Discussion}

We found that along the German Baltic coasts of Kiel Bight and Lübeck Bight the richness in both, species and functional groups, decreased slightly along the gradient of decreasing salinity (from NW to SE). Additionally, the relative abundance of heterotrophs (mostly Mytilus) increased in the same direction, what was accompanied by an increase of annual biomass production and a decrease of interannual variability of community composition. Much of this variance was found to be due to abundance changes of a few "driver" species, mainly Mytilus, Amphibalanus and Polysiphonia.

The abiotic variables with potentially the most important influence on recruitment and successional dynamics of shallow-water hard-bottom communities in the region we monitored are temperature, salinity, and current regime (e.g. Harley et al., 2006). pH as well as its shifts and fluctuations do not seem to have a major impact on Baltic biota (e.g. Havenhand, 2012). Temperature has effects on the physiological rates of adults, larvae and recruits as well as on phenology (e.g. Harley et al., 2006; O'Connor, 2007, Gardner et al., 2011; and Sommer et al., 2012). Salinity is a major structuring factor in the Baltic in general and also correlates closely with various community properties (e.g. composition, diversity, and productivity) (e.g. Ojaveer, 2010). Direction and velocity of currents are paramount for the transport of water masses, prey and propagules (e.g. BACC Author Group, 2008).The mean year-to-year dissimilarity of hydrographical summer conditions, i.e. current direction and velocity, SST (sea surface temperature) and salinity at the different stations from June to August, related positively to the

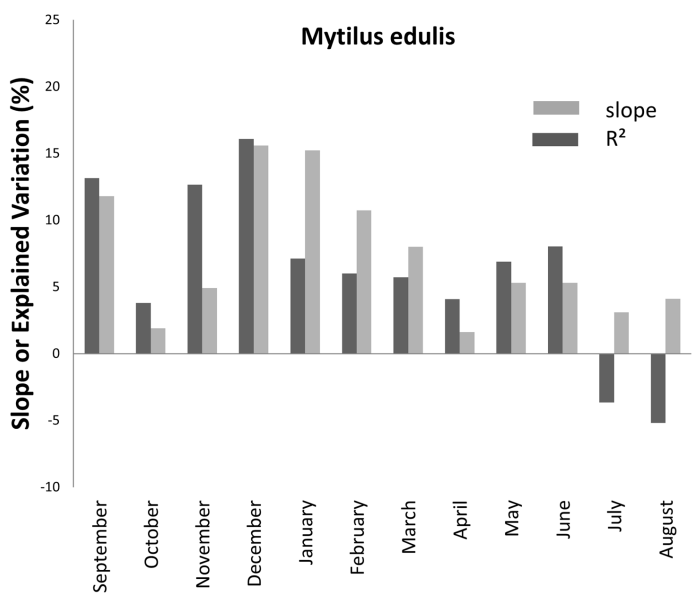

(B)

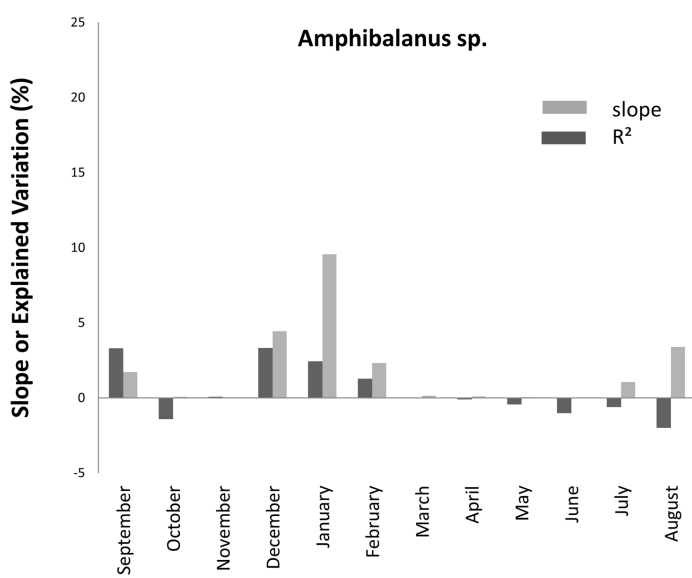

(C)

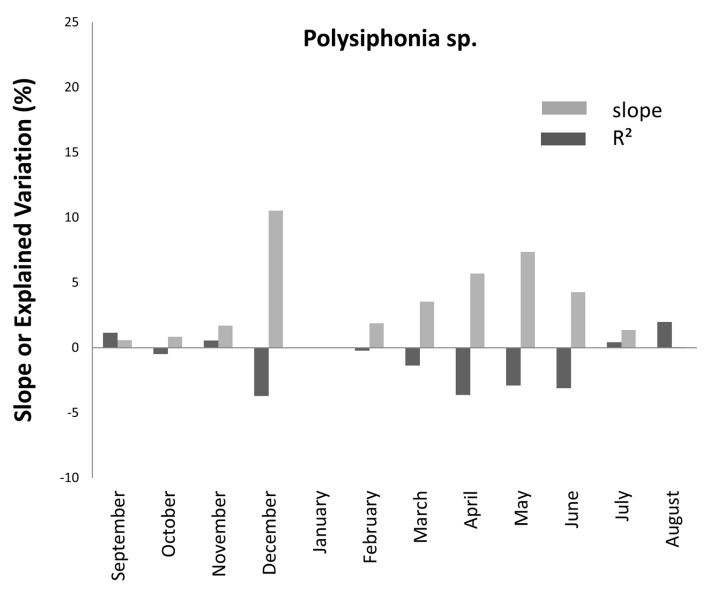

Fig. 13. Relationship (slopes and explained variance $R^{2}$ ) between monthly mean SST and the relative abundance of (A) Mytilus, (B) Amphibalanus and (C) Polysiphonia in the communities establishing on the panels. In case of Mytilus 4 of the relationships were significant at $p \geq 0.05$, while the number of significant findings was 1 in case of Amphibalanus and 0 for Polysiphonia. 
dissimilarity between years in the composition of communities. Abiotic variability explained $55 \%$ of the biological variability found during the five years of monitoring. However, breaking down "abiotic variability" into its components produced a diverse picture: salinity dissimilarity between consecutive years, which was on average between 4 and $6 \%$, did not contribute to the compositional dissimilarities measured. Long-term fluctuations in salinity, presumably, represented a minor stress to all those species which were primarily responsible for compositional variability, because none of them is, in the western Baltic, close to its lower salinity tolerance limit (e.g. Bonsdorff, 2006; and Schubert et al., 2011). All of these species occur at all stations that we sampled in the Kiel and Lübeck bights which are dissimilar with regard to salinity by up to $22 \%$. In contrast to this, temperature did affect the abundance of the aforementioned "driver" species which cause most of the structural change among consecutive years. The most common autotroph, Polysiphonia, for example, seemed to suffer from warm summers. For the time being, we do not know whether reproduction, growth or physiological performance of the algae were directly affected by warm conditions or whether detrimental interactions with parasites, pathogens, foulers, competitors or consumers intensified under warmer conditions (e.g. Harley et al., 2006; and Wahl et al., 2011b). Furthermore, the dominant heterotrophs, Amphibalanus and - particularly so - Mytilus, benefitted from warm winters. These species settle mainly in summer (June through August) and their larva are released 3-4 weeks prior to settlement. Hence, warmer winter temperatures might rather enhance the reproductive success of the parent population. At first glance this seems counterintuitive: warm winter temperatures should be stressful since they increase the metabolic rate of poikilotherms in times of low planktonic food availability. However, a warmer winter could also lead to earlier spring blooms (if not consumed by overwintering zooplankton; Sommer and Lewandowska, 2011) improving the food availability for mussels and barnacles in spring/early summer, i.e. when gonads maturate, gametes are released and larvae develop. The causal chain between mild winter temperatures and enhanced mussel and barnacle recruitment in the following may be indirect and tortuous - if it is not just a correlated covariance.

Interannual dissimilarity in the taxonomic composition of communities was closely followed by a dissimilarity in the composition of functional groups $\left(r^{2}=0.87, p=0.001\right)$. In this approach functional groups are defined by the combination of 4 functional traits describing a species' properties at the adult stage with regard to body size, growth form, trophic type and modularity. A difference in only one of these traits is sufficient to classify two functional groups as distinct in our analysis of dissimilarity. This linkage is justified if we assume that it makes a difference whether suspension feeding is carried out by a large, branching and colonial organism or a medium sized, solitary organism of massive growth form. The body size of solitary organisms has been regarded as a proxy for longevity and an indication of low metabolic rates, while growth form may determine competitiveness for resources including light and substratum (e.g. Woodward et al., 2005; Hillebrand, 2004; and Micheli and Halpern, 2005). When, however, we focus on the absence or presence of a certain service (e.g. bentho-pelagic coupling, primary production, and habitat engineering) in the community we may consider the traits singly, i.e. disregarding the other three traits of its bearer. Under this perspective, the substantial interannual variability in the composition of functional groups is not accompanied by a similar variability in the combination of ecological traits in the communities. In fact, essential services like primary production, suspension feeding or provision of structure were provided by at least one species in nearly all communities and by more than one species (i.e. redundantly) in the great majority of communities. Thus, as a general rule that follows from our analyses, despite a substantial interannual variability in functional group composition, the essential services of the shallow-water hard-bottom communities in the western Baltic were warranted. It should be noted at this point that by exclusively considering the sessile components of the assemblages on the settlement panels, the trophic diversity of animals was essentially reduced to one trait: suspension feeding.

Increased taxa diversity could strengthen the stability of community services because functional group richness approaches saturation in very taxa-rich communities. This pattern suggests that the more taxa rich a community is the higher is the probability that a functional group and, even more so, single functional traits are represented by more than one species. Consequently, the loss of a species will not automatically be accompanied by the loss of a function. This aspect of stability ("insurance hypothesis") cannot, however, be related directly to the interannual dissimilarity quantified here because each year the communities recruited anew from the meta-community of the region and without direct link to the previous year's panel community at a given site.

Our observation that interannual variability in the taxonomic composition of western Baltic hard-bottom communities decreases from NW to SE, which was paralleled by a shallow decrease in species richness, should not be interpreted as an inverse relationship between diversity and stability as shown by Valdivia and Molis (2009) for similar communities. First, the "disturbance" (replacement of the panels) was complete and its intensity unrelated to diversity. Second, the pattern of recruitment, or "recovery" from the disturbance was to a large extent influenced by the abiotic conditions, mainly currents and temperature, during the recruitment phase or prior to it (reproductive phase of the parent generation). If we consider the annual panel replacement as a patch disturbance within a larger meta-community and the recruitment onto the new panels over the following 12 months as patch re-colonization, we could ask whether taxonomically more diverse regions (i.e. gamma diversity of a station) show less variation in the recovery process even 
under variable abiotic conditions. It was postulated earlier that taxonomic diversity favours resistance to environmental change in functionally poor but not in functionally rich systems (Wahl et al., 2011a). This hypothesis, however, cannot be examined here, because the regions did not differ enough with regard to functional diversity.

In this study we have pursued a double aim: we attempted to quantify the temporal "noise" in the composition of oneyear old western Baltic hard-bottom communities, and we tried to identify some possible causes for the observed biotic variability. While the study has some strengths it also features certain limitations. Here we consider as strong points that the quantification of compositional variability was not confounded by community age, spatial variability, substratum type, sampling procedure, or season, all of which were kept constant. On the other hand, the recruitment and succession process was arrested at the age of 12 months and the interannual dissimilarity of communities could diverge or converge thereafter. Another limitation is that we only assessed sedentary species and ignored the variability of motile consumers. A further weakness will certainly decrease with the continuation of this project: while one of our major goals was to distinguish noise (natural fluctuations) from the signal of global change (directional shifts), we cannot be sure whether the signal is not already included in the noise. A signal would be detectable by an MDS trajectory (such as those shown in Fig. 9) over time that does not return to previous points. At this point in time, in only two cases, i.e. Boknis Eck and Staberhuk, we observed that the youngest point is furthest away from the oldest. It is well known, however, that climate change is overlain by local fluctuations and decadal oscillations (e.g. Parker et al., 2007; and Frid, 2011) and it is therefore advisable to assess communities' compositional variability on a decadal scale before tempting to quantify the range of "natural" background variability as the ecological noise. Also, this should only be undertaken, if the MDS trajectory does not indicate a tendency for directional change. Furthermore, the western Baltic Sea, the transition zone between brackish Baltic and saline North Sea waters, is known as an area of very high variability of oceanographic conditions (very noisy). Further to the east, in the Baltic roper, hydrographical conditions are more stable (less noisy) so that directional shifts due to climate change would be easier to detect. These points in mind, our results about natural noise in the composition of western Baltic hard-bottom communities should be considered with some caution while the ongoing program continuously solidifies this knowledge base.

The noise, i.e. the dissimilarity of community compositions between years, decreases from NW to SE along the decreasing diversity gradient and with the increasing relative abundance of heterotrophic organisms. While in Kiel Bight proper (stations $\mathrm{Fa}, \mathrm{B}, \mathrm{Sch}$ ) the communities vary structurally by, on average, $80 \%$ between years, this variability averages around only $55 \%$ in Lübeck Bight proper (stations $\mathrm{K}$ and $\mathrm{S}$ ). In addition, the confidence intervals of the inter- annual fluctuations cover the range between 75 and $93 \%$ in Kiel Bight and between 45 and $65 \%$ in Lübeck Bight. We assume that this apparently higher stability of community structure in the eastern stations is caused by reduced abiotic variability and the high relative abundance of the competitively extremely dominant mussel Mytilus edulis. A signal for a severe disturbance or a regime shift might be identified when the composition of communities recruited under the conditions described here leaves this confidence interval. Such a signal could be detected more easily in the less fluctuating communities of the eastern part of the salinity gradient studied here. Directional shift over time which might signal a change driven by climate change can be distinguished from a more random oscillation of community structure (or functional composition) using the RELATE procedure. This tool may prove useful to detect climate change signals when a time series of biological samples covering a decade or more is available. The close correlation between taxonomic and functional rhos in the RELATE analyses seems to indicate that any shift in species composition is associated with a shift in functional group composition. This is probably due to low redundancy of functional groups in this species-poor system. However, a shift in functional group composition is not necessarily associated with a loss (or gain) of functional traits or services in the community, as discussed above, but it bears the potential for such a loss or gain. It remains to be investigated whether RELATE is restricted to the detection of steady change in community composition or community functioning, and whether more abrupt shifts, i.e. abrupt regime shifts jeopardize its suitability.

We conclude that along the German Baltic coast of the Kiel and Lübeck bights the relative abundance of heterotrophs increases, annual productivity (biomass increment) tends to increase, and compositional variability in time tends to decrease. A substantial part of interannual compositional dissimilarity is driven by the variability of environmental conditions such as the current regime in summer and the temperatures in winter. Both of these are expected to change in the coming decades (BACC Author Group, 2008).

Acknowledgements. We greatly appreciate Renate Schuett's meticulous identification and quantification of the panel communities.

The service charges for this open access publication

have been covered by a Research Centre of the

Helmholtz Association.

Edited by: H. Bange

\section{References}

Anderson, D. M., Cembella, A. D., and Hallegraeff, G. M.: Progress in Understanding Harmful Algal Blooms: Paradigm Shifts and New Technologies for Research, Monitoring, and Management, Ann. Rev. Mar. Sci., 4, 143-176, 2012. 
BACC Author Group: Assessment of climate change for the Baltic Sea Basin, Springer, 2008.

Bonsdorff, E.: Zoobenthic diversity-gradients in the Baltic Sea: Continuous post-glacial succession in a stressed ecosystem, J. Exp. Mar. Biol. Ecol., 330, 383-391, 2006.

Bremner, J., Rogers, S. I., and Frid, C. L. J.: Matching biological traits to environmental conditions in marine benthic ecosystems, J. Mar. Syst., 60, 302-316, 2006a.

Bremner, J., Rogers, S. I., and Frid, C. L. J.: Methods for describing ecological functioning of marine benthic assemblages using biological traits analysis (BTA), Ecol. Indic., 6, 609-622, $2006 \mathrm{~b}$.

Bumke, K., Karger, U., Hasse, L., and Niekamp, K.: Evaporation over the Baltic Sea as an example of a semi-enclosed sea, Contr. Atmos. Phys., 71, 249-261, 1998.

Carr, M., Woodson, C. B., Cheriton, O. M., Malone, D., McManus, M. A., and Raimondi, P. T.: Knowledge through partnerships: integrating marine protected area monitoring and ocean observing systems, Front. Ecol. Environ., 9, 342-350, 2011.

Clarke, K. R. and Gorley, R. N.: PRIMER v6: User Manual/Tutorial, PRIMER-E, 2006.

Clarke, K. R. and Warwick, R. M.: Change in Marine Communities: An Approach to Statistical Analysis and Interpretation, PRIMER-E, 2001.

Cowie, J.: Climate change: biological and human impacts, University Press Cambridge, 487 pp., ISBN 978-0-521-69619-7, 2007.

Edwards, K. P., Hare, J. A., Werner, F. E., and Seim, H.: Using 2dimensional dispersal kernels to identify the dominant influences on larval dispersal on continental shelves, Mar. Ecol. Prog. Ser., 352, 77-87, 2007.

Firth, L. B. and Hawkins, S. J.: Introductory comments - Global change in marine ecosystems: Patterns, processes and interactions with regional and local scale impacts, J. Experim. Mar. Biol. Ecol., 400, 1-6, 2011.

Frid, C. L. J.: Temporal variability in the benthos: Does the sea floor function differently over time, J. Exp. Mar. Biol. Ecol., 400, 99107, 2011.

Frid, C. L. J., Buchanan, J. B., and Garwood, P. R.: Variability and stability in benthos:twenty-two years of monitoring off Northumberland, ICES J. Mar. Sci., 53, 978-980, 1996.

Frid, C. L. J., Paramor, O. A. L., Brockington, S., and Bremner, J.: Incorporating ecological functioning into the designation and management of marine protected areas, Hydrobiologia, 606, 6979, 2008.

Gamfeldt, L., Hillebrand, H., and Jonsson, P. R.: Multiple functions increase the importance of biodiversity for overall ecosystem functioning, Ecology, 89, 1223-1231, 2008.

Gardner, J. L., Peters, A., Kearney, M. R., Joseph, L., and Heinsohn, R.: Declining body size: a third universal response to warming, Trends Ecol. Evol., 26, 285-291, 2011.

Greene, C. H. and Schoener, A.: Succession on marine hard substrata - a fixed lottery, Oecologia, 55, 289-297, 1982.

Harley, C. D. G., Hughes, A. R., Hultgren, K. M., Miner, B. G., Sorte, C. J. B., Thornber, C. S., Rodriguez, L. F., Tomanek, L., and Williams, S. L.: The impacts of climate change in coastal marine systems, Ecol. Lett., 9, 228-241, 2006.

Havenhand, J. N.: How will Ocean Acidification Affect Baltic Sea Ecosystems? An Assessment of Plausible Impacts on Key Functional Groups, Ambio, 41, 637-644, 2012.
Hillebrand, H.: On the generality of the latitudinal diversity gradient, Am. Nat., 163, 192-211, 2004.

Hinrichsen, H.-H., Lehmann, A., St. John, M. S., and Brügge, B.: Modeling the cod larvae drift in the Bornholm Basin in summer 1994, Cont. Shelf Res., 17, 1765-1784, 1997.

Jackson, A. C. and McIlvenny, J.: Coastal squeeze on rocky shores in northern Scotland and some possible ecological impacts, J. Experim. Mar. Biol. Ecol., 400, 314-321, 2011.

Kordas, R. L., Harley, C. D. G., and O'Connor, M. I.: Community ecology in a warming world: The influence of temperature on interspecific interactions in marine systems, J. Experim. Mar. Biol. Ecol., 400, 218-226, 2011.

Lehmann, A. and Hinrichsen, H.-H.: On the thermohaline variability of the Baltic Sea, J. Mar. Syst., 25, 333-357, 2000.

Lehmann, A., Hinrichsen, H-H., and Krauss, W.: Effects of remote and local atmospheric forcing on circulation and upwelling in the Baltic Sea, Tellus, 54A, 299-316, 2002.

Lehmann, A., Getzlaff, K., and Harlaß, J.: Detailed assessment of climate variability in the Baltic Sea area for the period 1958 to 2009, Climate Res., 46, 185-196, 2011.

Lehmann, A., Myrberg, K., and Höflich, K.: A statistical approach to coastal upwelling in the Baltic Sea based on the analysis of satellite data for 1990-2009, Oceanologia, 54, 369-393, 2012.

Lockwood, B. L. and Somero, G. N.: Invasive and native blue mussels (genus Mytilus) on the California coast: The role of physiology in a biological invasion, J. Experim. Mar. Biol. Ecol., 400, 167-174, 2011

Loreau, M. and de Mazancourt, C.: Species synchrony and its drivers: Neutral and nonneutral community dynamics in fluctuating environments, American Naturalist, 172, E48-E66, 2008.

McNaughton, S. J.: Diversity and stability of ecological communities - comment on the role of empiricism in ecology, Am. Nat., 111, 515-525, 1977.

Merzouk, A. and Johnson, L. E.: Kelp distribution in the northwest Atlantic Ocean under a changing climate, Journal of Experimental Marine Biology and Ecology, 400, 90-98, 2011.

Micheli, F. and Halpern, B. S.: Low functional redundancy in coastal marine assemblages, Ecol. Lett., 8, 391-400, 2005.

Millennium Ecosystem Assessment: Ecosystems and human wellbeing: Synthesis, Island Press, 2005.

O'Connor, M. I., Bruno, J. F., Gaines, S. D., Halpern, B. S., Lester, S. E., Kinlan, B. P., and Weiss, J. M.: Temperature control of larval dispersal and the implications for marine ecology, evolution, and conservation, Proc. Natl. Acad. Sci. USA, 104, 1266-1271, 2007.

Ojaveer, H. A., Jaanus, B. R., MacKenzie, G. M., Olenin, S., Radziejewska, T., Telesh, I., Zettler, M. L., and Zaiko, A.: Status of Biodiversity in the Baltic Sea, Plos One, 5, e12467, doi:10.1371/journal.pone.0012467, 2010.

Parker, D., Folland, C., Scaife, A., Knight, J., Colman, A., Baines, P., and Dong, B. W.: Decadal to multidecadal variability and the climate change background, J. Geophys. Res.-Atmos., 112, D18115, doi:10.1029/2007JD008411, 2007.

Philippart, C. J. M., Anadon, R., Danovaro, R., Dippner, J. W., Drinkwater, K. F., Hawkins, S. J., Oguz, T., O'Sullivan, G. and Reid, P. C.: Impacts of climate change on European marine ecosystems: Observations, expectations and indicators, J. Experim. Mar. Biol. Ecol., 400, 52-69, 2011. 
Pollack, J. B., Palmer, T. A., and Montagna, P. A.: Long-term trends in the response of benthic macrofauna to climate variability in the Lavaca-Colorado Estuary, Texas, Mar. Ecol. Prog. Ser., 436, 67-80, 2011.

Preisendorfer, R. W.: Principal Component Analysis in Meteorology and Oceanography, Elsevier Science Publishers BV, New York, 1988.

R Development Core Team R: A language for statistical computing, R Foundation for Statistical Computing, Vienna, Austria, ISBN 3-900051-07-0, http://www.R-project.org, 2010.

Reise, K.: Mosaic cycles in the marine benthos, in: The mosaiccycle concept of ecosystems, edited by: Remmert, H., Springer, 1991.

Rudolph, C. and Lehmann, A.: A model-measurements comparison of atmospheric forcing and surface fluxes of the Baltic Sea, Oceanologia, 48, 333-380, 2006.

Schubert, H., Feuerpfeil, P., Marquardt, R., Telesh, I., and Skarlato, S.: Macroalgal diversity along the Baltic Sea salinity gradient challenges Remane's species-minimum concept, Mar. Poll. Bull., 62, 1948-1956, 2011.

Solan, M., Raffaelli, D. G., Paterson, D. M., White, P. C. L. and Pierce, G. J.: Marine biodiversity and ecosystem function: empirical approaches and future research needs - Introduction, Mar. Ecol.-Prog. Ser., 311, 175-178, 2006

Somero, G. N.: The physiology of climate change: how potentials for acclimatization and genetic adaptation will determine "winners" and "losers", J. Exp. Biol., 213, 912-920, 2010.

Sommer, U. and Lewandowska, A.: Climate change and the phytoplankton spring bloom: warming and overwintering zooplankton have similar effects on phytoplankton, Glob. Change Biol., 17, 154-162, 2011

Sommer, U., Aberle, N., Lengfellner, K., and Lewandowska, A.: The Baltic Sea spring phytoplankton bloom in a changing climate: an experimental approach, Mar. Biol., 159, 2479-2490, 2012.

Spencer, M., Birchenough, S. N. R., Mieszkowska, N., Robinson, L. A., Simpson, S. D., Burrows, M. T., Capasso, E., CleallHarding, P., Crummy, J., Duck, C., Eloire, D., Frost, M., Hall, A. J., Hawkins, S. J., Johns, D. G., Sims, D. W., Smyth, T. J. and Frid, C. L. J.: Temporal change in UK marine communities: trends or regime shifts?, Mar. Ecol. Evolut. Perspec., 32, 10-24, doi:10.1111/j.1439-0485.2010.00422.x, 2011
Thomsen, J., Saphörster, J., Heinemann, A., and Melzner, F.: Impacts of hypercapnia and temperature on physiological performance of marine invertebrates from the Baltic Sea, Comp. Biochem. Phys. A, 153A, S169-S169, 2009.

Thomsen, J., Gutowska, M. A., Saphörster, J., Heinemann, A., Trübenbach, K., Fietzke, J., Hiebenthal, C., Eisenhauer, A., Körtzinger, A., Wahl, M., and Melzner, F.: Calcifying invertebrates succeed in a naturally $\mathrm{CO}_{2}$-rich coastal habitat but are threatened by high levels of future acidification, Biogeosciences, 7, 3879-3891, doi:10.5194/bg-7-3879-2010, 2010.

Valdivia, N. and Molis, M.: Observational evidence of a negative biodiversity-stability relationship in intertidal epibenthic communities, Aquat. Biol., 4, 263-271, 2009.

von Bertalanffy, L.: The theory of open systems in physics and biology, Science, 111, 23-29, 1960.

Wahl, M.: Ecological modulation of environmental stress: interactions between ultraviolet radiation, epibiotic snail embryos, plants and herbivores, J. Animal Ecol., 77, 549-557, 2008

Wahl, M.: Habitat characteristics and typical functional groups, in: Marine Hard Bottom Communities: patterns, dynamics, diversity, and change, edited by: Wahl, M., Springer, 206, 7-18, 2009

Wahl, M., Link, H., Alexandridis, N., Thomason, J. C., Cifuentes, M., Costello, M. J., da Gama, B. A. P., Hillock, K., Hobday, A. J., Kaufmann, M. J., Keller, S., Kraufvelin, P., Krüger, I., Lauterbach, L., Antunes, B .L., Molis, M., Nakaoka, M., Nyström, J., Radzi, Z., Stockhausen, B., Thiel, M., Vance, T., Weseloh, A., Whittle, M., Wiesmann, L., Wunderer, L., Yamakita, T., and Lenz, M.: Re-structuring of marine communities exposed environmental change: A global study on the interactive effects of species and functional richness, Plos One, 6, e19514, doi:10.1371/journal.pone.0019514, 2011a.

Wahl, M., Jormalainen, V., Eriksson, B. K., Coyer, J. A., Molis, M., Schubert, H., Dethier, M., Karez, R., Kruse, I., Lenz, M., Pearson, G., Rohde, S., Wikström, S. A., and Olsen, J. L.: Stress ecology in Fucus: abiotic, biotic and genetic interactions, Adv. Mar. Biol., 59, 37-105, 2011b.

Woodward, G.,Ebenman, B., Emmerson, M., Montoya, J. M., Olesen, J. M., Valido, A., and Warren, P. H.: Body size in ecological networks, Trends Ecol. Evol., 20, 402-409, 2005.

Yachi, S. and Loreau, M.: Biodiversity and ecosystem productivity in a fluctuating environment: the insurance hypothesis, Proc. Natl. Acad. Sci. USA, 96, 1463-1468, 1999. 